\title{
Tyrosinase-crosslinked, tissue adhesive and biomimetic alginate sulfate hydrogels for cartilage repair
}

\section{Journal Article}

Author(s):

Öztürk, Ece; Stauber, Tino (D); Levinson, Clara; Cavalli, Emma; Arlov, Øystein; Zenobi-Wong, Marcy (D)

Publication date:

2020-07

Permanent link:

https://doi.org/10.3929/ethz-b-000430712

Rights / license:

$\underline{\text { In Copyright - Non-Commercial Use Permitted }}$

Originally published in:

Biomedical Materials 15(4), https://doi.org/10.1088/1748-605X/ab8318

\section{Funding acknowledgement:}

159783 - A Bio-inspired Cartilage Mimetic with Enhanced Chondrogenic and Anti-inflammatory Properties (SNF)

143667 - Drug-induced expression of the chondrogenic phenotype using a nanofiber-reinforced cartilage mimetic (SNF) 


\section{Tyrosinase-crosslinked, tissue adhesive and biomimetic alginate sulfate hydrogels for cartilage repair}

Ece Öztürk ${ }^{1,2,3}$, Tino Stauber ${ }^{1}$, Clara Levinson ${ }^{1}$, Emma Cavalli ${ }^{1}$, Øystein Arlov ${ }^{4}$ and Marcy Zenobi-Wong ${ }^{1, *}$

${ }^{1}$ Tissue Engineering and Biofabrication Laboratory, ETH Zurich, Otto-Stern-Weg 7, 8093 Zurich, Switzerland

${ }^{2}$ Koç University, School of Medicine, Rumelifeneri Yolu, Istanbul, 34450, Turkey

${ }^{3}$ Koç University, Research Center for Translational Medicine, Rumelifeneri Yolu, Istanbul, 34450, Turkey

${ }^{4}$ Department of Biotechnology and Nanomedicine, SINTEF Industry, Richard Birkelands vei 3B, 7034 Trondheim, Norway

* Corresponding author

KEYWORDS: Cartilage engineering, biomimetic materials, tissue adhesive

\section{ABSTRACT}

The native cartilage extracellular matrix (ECM) is enriched in sulfated glycosaminoglycans with important roles in the signaling and phenotype of resident chondrocytes. Recapitulating the key ECM components within engineered tissues through biomimicking strategies has potential to improve the regenerative capacity of encapsulated cells and lead to better clinical outcome. Here, we developed a double-modified, biomimetic and tissue adhesive hydrogel for cartilage 
engineering. We demonstrated sequential modification of alginate with first sulfate moieties to mimic the high glycosaminoglycan content of native cartilage and then tyramine moieties to allow in situ enzymatic crosslinking with tyrosinase under physiological conditions. Tyrosinasecrosslinked alginate sulfate tyramine (ASTA) hydrogels showed strong adhesion to native cartilage tissue with higher bond strength compared to alginate tyramine (AlgTA). Both ASTA and AlgTA hydrogels supported the viability of encapsulated bovine chondrocytes and induced a strong increase in the expression of chondrogenic genes such as collagen 2, aggrecan and Sox9. Aggrecan and Sox9 gene expression of chondrocytes in ASTA hydrogels were significantly higher than those in AlgTA. Chondrocytes in both ASTA and AlgTA hydrogels showed potent deposition of cartilage matrix components collagen 2 and aggrecan after 3 weeks of culture whereas a decreased collagen 1 deposition was observed in the sulfated hydrogels. ASTA and AlgTA hydrogels with encapsulated human chondrocytes showed in vivo stability as well as cartilage matrix deposition upon subcutaneous implantation into mice for 4 weeks. Our data is the first demonstration of a double-modified alginate with sulfation and tyramination that allows in situ enzymatic crosslinking, strong adhesion to native cartilage and chondrogenic re-differentiation.

\section{INTRODUCTION}

Cartilage tissue has a limited capacity for self-regeneration due to its avascular structure. Thus, cartilage injuries upon acute trauma or degenerative pathologies lead to severe joint pain and disabilities. Tissue engineering approaches have been implemented to cartilage repair with methods such as autologous chondrocyte implantation (ACI) (1). However, the limited number of autologous chondrocytes obtained from cartilage biopsies demand two-dimensional (2D) expansion of cells which leads to their de-differentiation and loss of phenotype (2). Native cartilage is a three-dimensional (3D) and highly hydrated microenvironment comprised mainly of a network 
of collagen 2 fibers and proteoglycans such as aggrecan (3). Encapsulation of chondrocytes into 3D culturing environments such as hydrogels of biological or synthetic origin has been extensively shown to induce re-differentiation of chondrocytes and expression of cartilage-specific matrix components (4-6). Matrix-assisted autologous chondrocyte implantation (MACI) has been developed to implant chondrocytes embedded in a 3D microenvironment for induction of chondrocyte phenotype and improving regeneration of hyaline cartilage tissue (1). Implantation of preformed hydrogels has disadvantages such as two-step and invasive surgical procedures and necessity of fitting the materials into defects with irregular shapes. Injectable, in situ forming hydrogels have emerged in the recent years to overcome these disadvantages and have been shown to effectively co-deliver cells and bioactive molecules in a minimally invasive and targeted manner (7-9). Enzymatic crosslinking of polymer networks for in situ hydrogel formation have gained attention due to their physiological and cell-compatible reaction conditions and substrate specificity (10). Naturally-derived enzymes such as transglutaminase and horseradish peroxidase (HRP) have been used to crosslink various polymers for cartilage tissue engineering (10-13). HRP catalyzes hydrogel formation of biopolymers modified with hydroxyphenyl-containing moieties such as tyramine in the presence of hydrogen peroxide $\left(\mathrm{H}_{2} \mathrm{O}_{2}\right)$. Furthermore, hydroxyphenyl groups are present in the native extracellular matrices (ECM) such as tyrosine residues rendering peroxidase-mediated adhesion of these tyramine-modified biopolymers to tissues possible (10, 14). Adhesion of engineered hydrogels to the defect site is crucial for their integration into the surrounding cartilage tissue and their stability (10). Tyrosinases belong to another family of naturally occurring enzymes called phenol oxidases and can catalyze the bonding of hydroxyphenyl groups in the presence of oxygen (10). Therefore, similar to peroxidases, tyrosinases have been used for conjugation of synthetic or biological polymers modified with 
phenolic moieties such as 3,4-dihydroxyphenylalanin (DOPA) or tyramine, grafting of proteins onto polymer backbones and adhesive properties (15-21). Recently, tyrosinase-crosslinking have gained interest in tissue engineering context and been shown to allow dynamic hydrogels with tunability of tissue mechanics and adhesiveness (22-25).

An important requirement for effective tissue engineering scaffolds is to provide cellular cues that mimic the native tissue microenvironment. Cartilage tissue is enriched in proteoglycans which are grafted with sulfated glycosaminoglycans (GAGs). Sulfated GAGs both contribute to the compressive strength of the tissue through high entrapment of water and to signaling of chondrocytes through mediation of growth factor interactions with their receptors (3). We have recently shown that biomimicking the native characteristics of cartilage tissue through sulfation of alginate drives mitogenicity of chondrocytes, promotes cartilage matrix production and prevents chondrocyte de-differentiation via induction of fibroblastic growth factor (FGF) signaling in a GAG-mimicking manner (26). However, ionically-crosslinked alginate sulfate hydrogels were mechanically not stable in vivo unless they were reinforced with a network of electrospun poly ( $\varepsilon$ caprolactone) (PCL) fibers (27).

In this work, we developed a biomimetic, tissue adhesive and stable hydrogel for cartilage tissue engineering. We performed double modification on alginate with sulfate and tyramine moieties that would enable enzymatic covalent crosslinking in the presence of tyrosinase. Alginate sulfate tyramine (ASTA) hydrogels support chondrocyte viability and re-differentiation and demonstrate strong adhesion to native cartilage as well as in vivo stability upon subcutaneous implantation indicating a promising potential for use in cartilage repair.

\section{RESULTS AND DISCUSSION}




\subsection{Synthesis and characterization of AlgTA and ASTA hydrogels}

Sulfation of alginate was performed as previously reported for modification of hydroxyl groups in alginate monomers with sulfate moieties via reacting with chlorosulfonic acid $\left(\mathrm{HClSO}_{3}\right)($ Figure 1(a)). The reaction has been shown to yield tunable sulfation of alginates by varying the $\mathrm{HClSO}_{3}$ concentration (28). Here, we used $2 \% \mathrm{v} / \mathrm{v} \mathrm{HClSO}_{3}$ for the sulfation reaction which led to a final sulfur content of $51.2 \mu \mathrm{g} / \mathrm{mg}$ shown by elemental analysis that corresponded to a degree of sulfation (DS) of 0.44 sulfate groups per monosaccharide (Figure 2(a)). A small decrease in the average molecular weight and molecular number average of alginate was observed with sulfation due to acid hydrolysis (Figure 2(b)). However, the uniformity of the chain length given by the dispersity index values did not differ significantly.

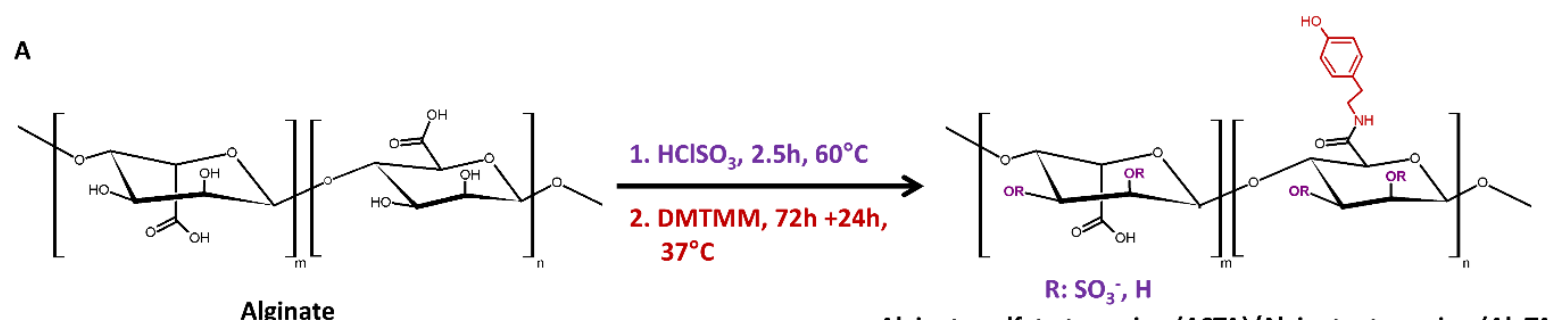

B

Alginate sulfate tyramine (ASTA)/Alginate tyramine (AlgTA)
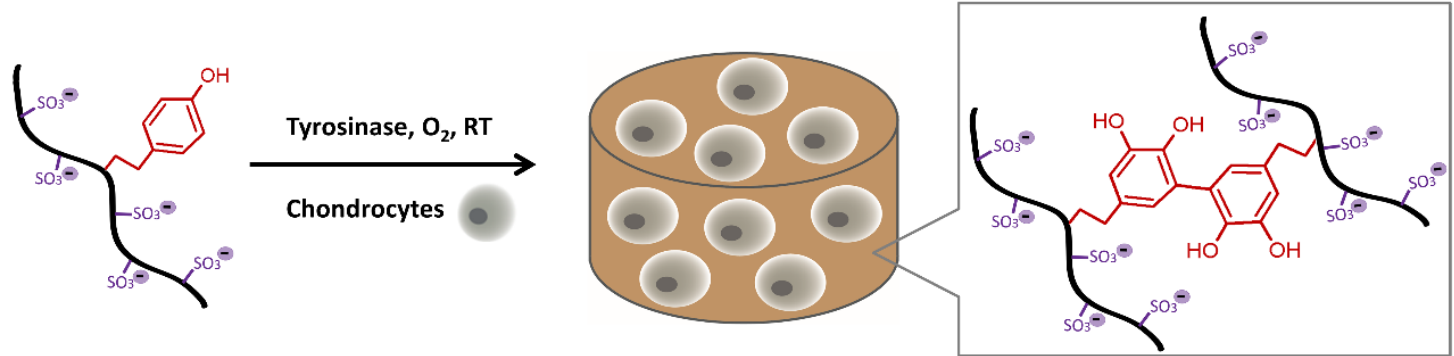

Figure 1: Synthesis of alginate tyramine (AlgTA) and alginate sulfate tyramine (ASTA). (a) Scheme showing the sulfation and subsequent tyramine modification of alginate. (b) Depiction of hydrogel formation of AlgTA and ASTA in the presence of tyrosinase via formation of di-tyramine bonds and encapsulation of chondrocytes. 
Tyramine conjugation to alginate and alginate sulfate samples was carried out via amidation of carboxyl groups using 4-(4,6-dimethoxy-1,3,5-triazin-2-yl)-4-methylmorpholiniumchloride (DMTMM) as the coupling agent. DMTMM has been recently shown for successful tyramine modification of hyaluronan (29). We confirmed the presence of tyramines with ${ }^{1} \mathrm{H}$ NMR spectroscopy by the appearance of aromatic peaks at 6.85 and $7.2 \mathrm{ppm}$ in line with previous reports (30). A third aromatic peak was observed at $7.4 \mathrm{ppm}$ (Figure 2(c)) in both AlgTA and ASTA samples. The same peak was reported for tyramine conjugation to hyaluronan via DMTMM due to formation of Tyr-O-DMT adducts from the excess of DMTMM (29). The molar degree of substitution (DSmol, \%) was calculated with UV-vis spectroscopy. We achieved very high DSmol values of $33.5 \pm 2.8$ for AlgTA and $31.7 \pm 1.8$ for the ASTA sample by performing two subsequent rounds of tyramine modification (Figure 2(d)). On the other hand, the reduction in the molecular weight of alginate due to sulfation did not cause a significant change in the extent of tyramine modification as AlgTA and ASTA samples yielded similar DSmol values.

AlgTA and ASTA hydrogels were formed by mixing the tyramine-modified polymers with tyrosinase at $\mathrm{pH} 7.4$ (Figure 1(b)). Tyrosinase catalyzes the formation of activated $o$-quinones from the phenol group of tyramine in the presence of oxygen. Activated quinones can react with each other to form di-tyramine bonds or to amino groups via Michael type addition $(10,31)$. Only a few studies addressed tyrosinase-crosslinked networks of polymers. Hydrogels of gelatin and chitosan crosslinked with tyrosinase has been reported with faster gelation when compared to transglutaminase $(15,16)$. However, hydrogels only formed with an amine-containing backbone such as chitosan in the presence of tyrosinase. Furthermore, tyrosinase-crosslinked hydrogels were shown to be mechanically weaker with fast degradation compared to hydrogels formed with transglutaminase (16). Tyrosinase-mediated conjugation of silk fibroin and chitosan was also 
reported yielding adhesive and non-toxic conjugates (19). Tyrosinase was shown to be efficient in crosslinking of proteins to polysaccharides such as conjugation of sericin onto chitosan (17). Hydrogels of tyramine-modified chondroitin sulfate crosslinked with tyrosinase have been shown to support growth of fibroblasts (32). Tyrosinase-mediated crosslinking of silk fibroin and gelatin was reported for differentiation of mesenchymal stem cells in bioprintable scaffolds (33). Recently, tyrosinase crosslinking was shown to yield dynamic hydrogels that allow tunability of tissue stiffness for modeling tumor tissue stiffening (24). Furthermore, tissue adhesive tyrosinasecrosslinked hydrogels have been demonstrated for use in meniscal repair (22).

Here, we demonstrate for the first time tyrosinase-crosslinked hydrogel networks of doublemodified alginate with sulfate and tyramine groups. AlgTA and ASTA solutions led to stable hydrogels under $10 \mathrm{~min}$ in the presence of tyrosinase (Figure 2(e)). Although there was not a significant difference in the degree of tyramine modification of the two polymers, AlgTA was used at a polymer concentration of $1 \% \mathrm{w} / \mathrm{v}$ whereas $2 \% \mathrm{w} / \mathrm{v}$ was used for ASTA to match the mechanical properties of the hydrogels possibly due to differences in their molecular weight. The compressive moduli of AlgTA ( $1 \% \mathrm{w} / \mathrm{v})$ and ASTA ( $2 \% \mathrm{w} / \mathrm{v})$ hydrogels were found to be $7.18 \pm$ $1.1 \mathrm{kPa}$ and $7.58 \pm 0.33 \mathrm{kPa}$ respectively and no significant difference was detected between the samples (Figure 2(f)). The hydrogels were also tested for their swelling behavior. Ionicallycrosslinked hydrogels of alginate sulfate have been shown to exhibit greater swelling compared to alginate hydrogels (26). On the contrary, tyrosinase crosslinked AlgTA and ASTA hydrogels demonstrated similar percent mass swelling (Figure 2(g)). 
A

\begin{tabular}{c|c|c}
\hline Sample & $\begin{array}{c}\text { Sulfur content } \\
(\mu \mathrm{g} / \mathrm{mg})\end{array}$ & $\begin{array}{c}\text { DS } \\
\text { (Sulfates per } \text { monomer) }\end{array}$ \\
\hline AlgTA & 0.16 & 0.01 \\
\hline ASTA & 51.2 & 0.44 \\
\hline
\end{tabular}

B

\begin{tabular}{c|c|c|c}
\hline Sample & Mn (kDa) & Mw (kDa) & $\begin{array}{c}\text { Dispersity index } \\
(\mathbf{M w} / \mathbf{M n})\end{array}$ \\
\hline AlgTA & $129.1 \pm 20.5$ & $279.8 \pm 4.3$ & $2.2 \pm 0.32$ \\
\hline ASTA & $119.5 \pm 5.6$ & $226.7 \pm 2.3$ & $1.9 \pm 0.10$ \\
\hline
\end{tabular}

D

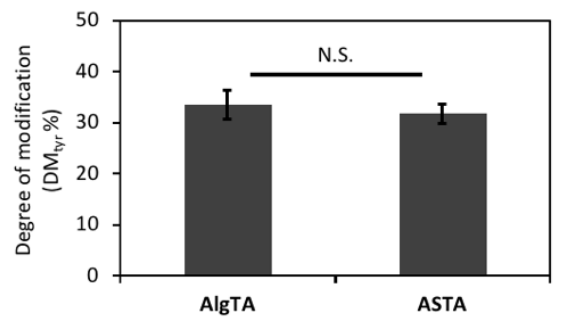

$\mathbf{E}$

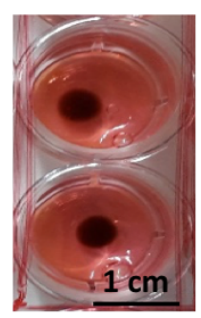

C

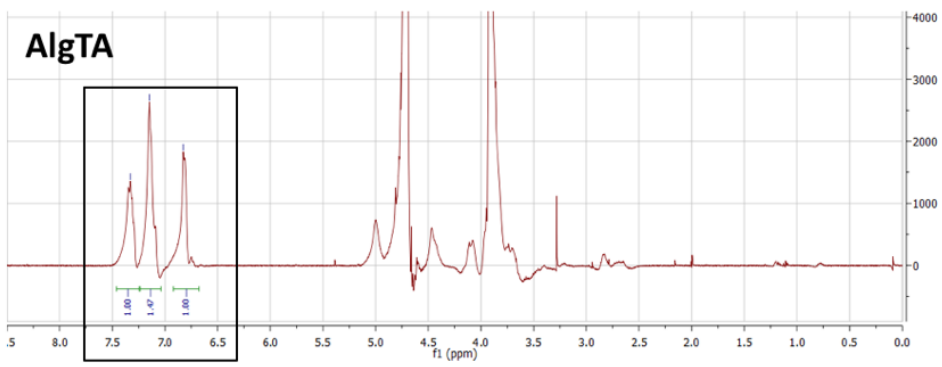

ASTA

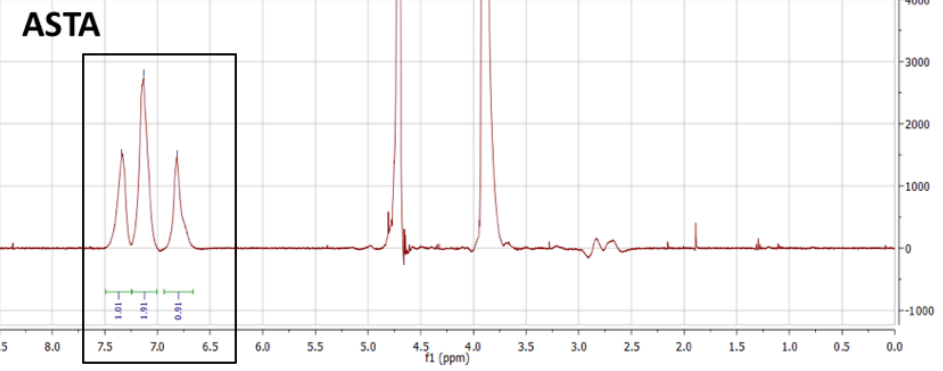

G

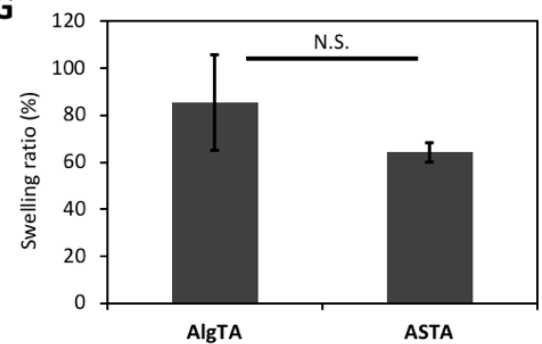

Figure 2: Characterization of alginate tyramine (AlgTA) and alginate sulfate tyramine (ASTA).

(a) Sulfur content in the alginate and alginate sulfate samples determined by elemental analysis with HR-ICP-MS and degree of sulfation (DS) expressed as the number of sulfate groups per monomer. (b) Molecular weight average $\left(\mathrm{M}_{\mathrm{W}}\right)$, molecular number average $\left(\mathrm{M}_{\mathrm{N}}\right)$ and the dispersity index $\left(\mathrm{M}_{\mathrm{W}} / \mathrm{M}_{\mathrm{N}}\right)$ values of alginate and alginate sulfate samples determined by SEC-MALLS. A refractive index $(\mathrm{dn} / \mathrm{dc})$ of 0.15 was used for all the samples. (c) ${ }^{1} \mathrm{H}$ NMR spectra $\left(\mathrm{D}_{2} \mathrm{O}\right)$ of $\operatorname{AlgTA}$ and ASTA polymers, appearance of aromatic peaks of tyramine was indicated with the black boxes. (d) Degree of tyramine modification in the AlgTA and ASTA samples (DSmol, \%, molar ratio of conjugated tyramine residues to the total number of carboxyl groups in alginate) determined by UV-vis spectroscopy. (e) Macroscopic appearance of AlgTA (top) and ASTA (bottom) hydrogels crosslinked with tyrosinase. (f) Compressive modulus of AlgTA and ASTA 
hydrogels shown as mean \pm s.d. for $\mathrm{n}=3$. (g) Percent mass swelling of AlgTA and ASTA hydrogels after 48 hours. Data is expressed as mean \pm s.d. for $n=5$, N.S.: nonsignificant.

\subsection{Adhesive properties of AIgTA and ASTA hydrogels to cartilage tissue}

Adhesion of engineered scaffolds to cartilage tissue is important for tissue integration in the defect site and long-term stability. With this aim, tissue adhesives such as fibrin glue have been frequently used in surgical cartilage repair $(34,35)$. Engineered tissue adhesives for cartilage have been also developed from biomaterials such as chondroitin sulfate and shown to efficiently mediate integration of polymeric scaffolds to cartilage defects (36) and induce in vivo regeneration (37). Tyramine-modified dextran and hyaluronan hydrogels crosslinked with HRP and $\mathrm{H}_{2} \mathrm{O}_{2}$ were demonstrated to have cartilage adhesive properties superior than fibrin glue $(10,14)$. Although tyrosinase was shown to exert water-resistant adhesive properties to biopolymers such as chitosan via oxidation of DOPA moieties, adhesion to native cartilage has not yet been addressed (21).

Alginate is an inert biopolymer that lacks cell adhesion moieties as well as tissue adhesiveness (38). However, modification of alginate such as networks of oxidized alginate and gelatin formed in the presence of borax via Schiff's reaction was shown to exert adhesiveness and integration to cartilage tissue (39). Here, we investigated the adhesive properties of in situ crosslinked AlgTA and ASTA hydrogels with tyrosinase to native cartilage tissue. We hypothesized that tyrosinase would mediate conjugation of tyramines of the biopolymer backbone to the tyrosine residues in the collagen network of cartilage. Our set-up comprised of a push-out test where hydrogels were directly casted in cartilage rings and pushed by a metal rod (Figure 3(a)). Cartilage rings were initially treated with chondroitinase $(1 \mathrm{U} / \mathrm{mL})$ which has previously been shown to yield better exposure of collagens (13). AlgTA and ASTA demonstrated strong adhesion to cartilage rings 
with in situ tyrosinase crosslinking. After the push-out test, we observed that the bulk of the gel was broken whereas the hydrogel-cartilage interface was still intact (Figure 3(b)). This was the case for both AlgTA and ASTA as we did not observe any visual differences between the samples in the push-out test. Then, we compared the bond strength achieved with tyrosinase crosslinking to the bond strength obtained from calcium crosslinking of the same biopolymers. Tyrosinase crosslinking led to a potent increase in bond strength of both AlgTA and ASTA hydrogels compared to calcium crosslinking. However, the bond strength achieved with ASTA hydrogels was up to $10 \pm 4 \mathrm{kPa}$ and found to be higher than AlgTA (Figure 3(c)). The difference in the adhesion strength of the two hydrogels could possibly be explained by the differences in the polymer content in the hydrogels. Furthermore, ASTA has slightly lower molecular weight compared to AlgTA (Figure 2(b)). Lower molecular weight polymer chains could exert higher mobility and lead to more contact points at the interaction site between the polymer and surface. Molecular weight has been shown to have an effect on the adhesive strength of mussel-mimetic DOPA-modified polymers in the presence of $\mathrm{IO}_{4}$ as the crosslinker (40). 


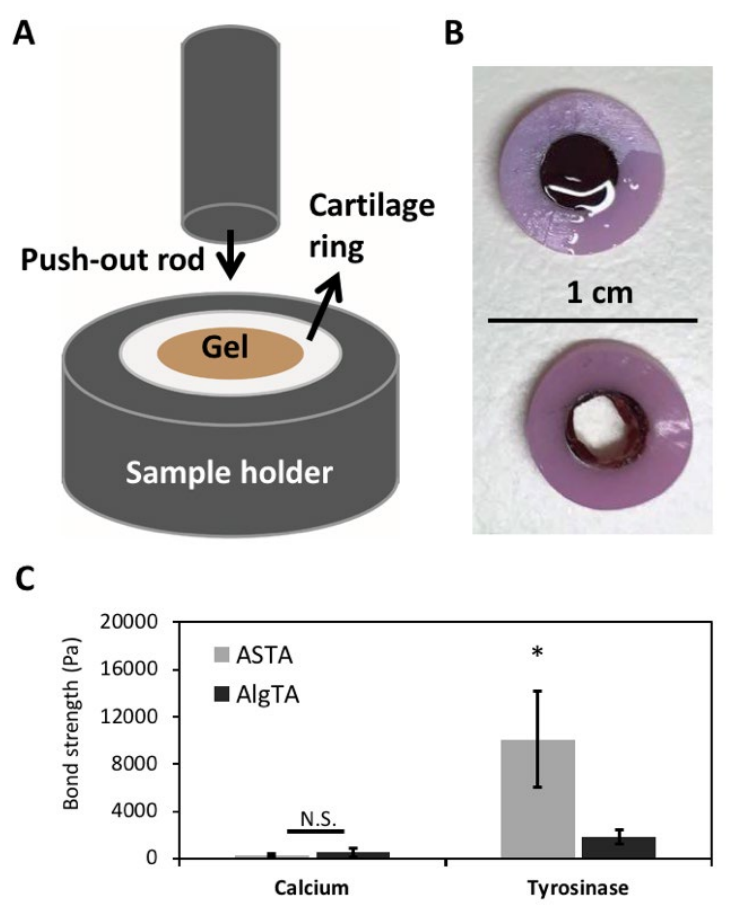

Figure 3: Adhesion of alginate tyramine (AlgTA) and alginate sulfate tyramine (ASTA) to cartilage tissue. (a) Scheme showing the set-up of the push-out test. A push-out rod with a diameter of $3 \mathrm{~mm}$ was used to push AlgTA or ASTA hydrogels casted in a cartilage ring (outer diameter: 8 $\mathrm{mm}$, inner diameter: $4 \mathrm{~mm}$ ). (b) Macroscopic images of an ASTA hydrogel casted in a cartilage ring before (top) and after (bottom) the push-out test. (c) Bond strength of AlgTA and ASTA hydrogels crosslinked with tyrosinase $(1 \mathrm{kU} / \mathrm{ml})$ or $\mathrm{CaCl}_{2}(100 \mathrm{mM})$. Data is expressed as mean \pm s.d. for $\mathrm{n}=3$; *: $\mathrm{p}<0.05$ when compared to ASTA crosslinked with calcium, N.S.: nonsignificant.

\subsection{In vitro analysis of AIgTA and ASTA hydrogels for chondrocyte growth and re- differentiation}

Next, we investigated chondrocyte growth and chondrogenic re-differentiation in AlgTA and ASTA hydrogels. We encapsulated primary chondrocytes in AlgTA and ASTA hydrogels and cultured them in chondrogenic medium containing TGF- $\beta 3$ to induce re-expression of chondrogenic markers. Chondrocyte viability was assessed with live/dead staining after 21 days 
of culture. Chondrocytes demonstrated overall good viability in both AlgTA and ASTA hydrogels (Figure 4(a)). Quantification of viability showed that there were no statistically significant differences between AlgTA and ASTA hydrogels (Figure 4(b)).

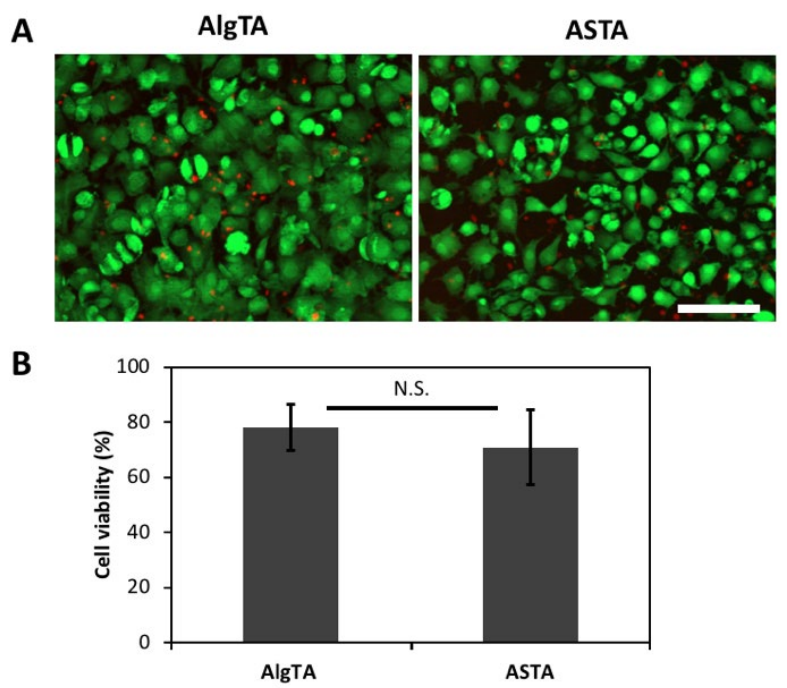

Figure 4: Chondrocyte viability in alginate tyramine (AlgTA) and alginate sulfate tyramine (ASTA) hydrogels. (a) Live/dead images showing live cells stained with calcein AM (green) and dead cells stained with propidium iodide (red) in AlgTA and ASTA hydrogels crosslinked with tyrosinase $(1 \mathrm{kU} / \mathrm{mL})$ and cultured in chondrogenic medium for 21 days. Scale bar: $100 \mu \mathrm{m}$. (b) Quantification of cell viability in hydrogels expressed as mean \pm s.d. for $n=3$, N.S.: nonsignificant. Then, we performed gene expression analysis of chondrocytes cultured in AlgTA and ASTA hydrogels and induced with TGF- $\beta 3$-containing chondrogenic medium for 21 days. We explored the expression of cartilage-specific markers collagen2, aggrecan and Sox9 in both hydrogels and compared to the gene expression of chondrocytes grown on 2D tissue culture plastic. Chondrocytes demonstrated a strong upregulation of collagen 2 in both AlgTA and ASTA hydrogels which was significantly higher than chondrocytes cultured on 2D (Figure 5(a)). Collagen 2 expression was higher in chondrocytes encapsulated in AlgTA hydrogels compared to ASTA. Aggrecan 
expression was also induced in chondrocytes encapsulated in hydrogels with a significantly higher expression compared to 2D culturing. Interestingly, chondrocytes in ASTA hydrogels exhibited a significantly higher expression of aggrecan than chondrocytes in AlgTA. Similarly, Sox9 expression was potently upregulated when chondrocytes were encapsulated in AlgTA and ASTA hydrogels compared to 2D. Sox9 expression in ASTA hydrogels was also found to be significantly higher than in AlgTA (Figure 5(a)). We investigated the expression of de-differentiation marker collagen 1 and catabolic markers ADAMTS5 and MMP13. We have previously shown that sulfation in the microenvironment exerted chondroprotective effects on chondrocytes through mediation FGF signaling in a heparin-mimetic manner (26). We reported that encapsulation of freshly-isolated chondrocytes in ionically-crosslinked alginate sulfate hydrogels led to a suppression of collagen 1, ADAMTS5 and MMP13 expression compared to hydrogels of unmodified alginate (26). Here, we did not detect a change in the expression of collagen 1 in chondrocytes encapsulated in AlgTA and ASTA hydrogels when compared to chondrocytes cultured on 2D or to each other (Figure 5(b)). On the other hand, chondrocytes showed a strong downregulation of ADAMTS5 in both hydrogels. Expression of MMP13 did not change in response to encapsulation in AlgTA hydrogels compared to 2D whereas a significant upregulation was observed in ASTA hydrogels compared to both AlgTA and 2D. Such upregulation of MMP13 contradicts our previous results where sulfated alginate hydrogels inhibited MMP13 in freshlyisolated chondrocytes via FGF signaling (26). The differences in these results could be affected by several factors including the different crosslinking of hydrogels, using of passaged chondrocytes and different culturing medium. In this study, we used serum-free chondrogenic medium supplemented with TGF- $\beta 3$ to induce re-differentiation of passaged chondrocytes. TGF- $\beta$ signaling has been shown to be a potent inducer of MMP13 expression mediated by activation of 
downstream effectors such as Smad3 (41) and p38 mitogen-activated protein kinase (MAPK) (42). Therefore, as opposed to chondroprotective effects of FGFs in serum-containing medium, induction of TGF- $\beta$ signaling might have led to induction of MMP13 expression. Sulfated alginate has been shown to be a heparin-mimetic and exert high affinity to several heparin-binding growth factors including TGF- $\beta$ family $(28,43)$. Therefore, such enhancement of TGF- $\beta$ signaling might have accounted for the aforementioned effects on MMP13 expression observed in chondrocytes.
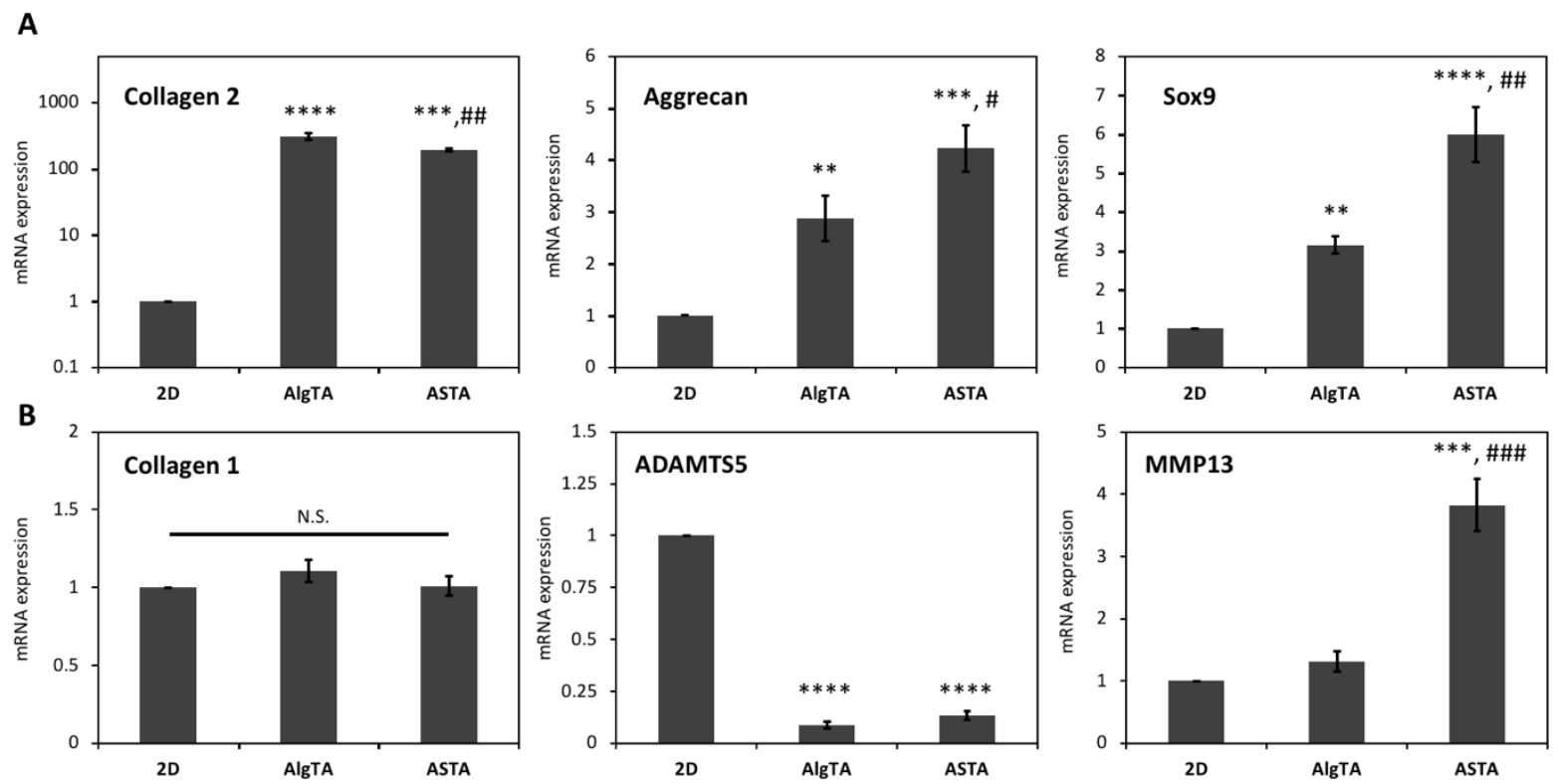

Figure 5: Chondrogenic re-differentiation in alginate tyramine (AlgTA) and alginate sulfate tyramine (ASTA) hydrogels. (a) mRNA expression of chondrogenic markers collagen 2, aggrecan and Sox9 in chondrocytes encapsulated in AlgTA and ASTA hydrogels crosslinked with tyrosinase $(1 \mathrm{kU} / \mathrm{mL})$ and cultured for 21 days in chondrogenic medium. (b) mRNA expression of de-differentiation and catabolic markers collagen 1, ADAMTS5 and MMP13 in chondrocytes. Data was normalized to chondrocytes cultured on tissue culture plastic (2D) in growth medium and expressed as mean \pm s.d. for $\mathrm{n}=3 .{ }^{* *}: \mathrm{p}<0.01,{ }^{* * *}: \mathrm{p}<0.001,{ }^{* * * *}: \mathrm{p}<0.0001$ when compared to $2 \mathrm{D}, \#: \mathrm{p}<0.05, \# \#: \mathrm{p}<0.01, \# \#: \mathrm{p}<0.001$ when compared to AlgTA, N.S.: nonsignificant. 
We next assessed production of cartilage matrix components collagen 2 and aggrecan in chondrocytes encapsulated in AlgTA and ASTA hydrogels. Both AlgTA and ASTA hydrogels supported potent deposition of collagen 2 after 21 days (Figure 6(a), Figure S1). This collagen 2 deposition was dependent on inducing the chondrocytes with chondrogenic media containing TGF- $\beta 3$ as collagen 2 deposition was found to be minimal when chondrocytes were cultured in basal medium without any TGF- $\beta 3$ (Figure S2). In line with gene expression data, chondrocytes encapsulated in ASTA hydrogels demonstrated enhanced deposition of aggrecan compared to AlgTA. We recently reported that aggrecan deposition of chondrocytes increased in a doseresponsive manner to the degree of sulfation in the microenvironment whereas collagen 1 deposition showed a decrease (26). Consistently, we observed less deposition of collagen 1 in chondrocytes encapsulated in ASTA hydrogels than AlgTA (Figure 6(a)). We also assessed the mechanical properties of hydrogels after culturing with chondrocytes for 21 days. Both hydrogels showed an increase when compared to the compressive moduli of the acellular gels indicating the effect of in vitro matrix formation. AlgTA hydrogels had a slightly higher compressive modulus than ASTA hydrogels; however, there was not a significant difference (Figure 6(b)). 
A
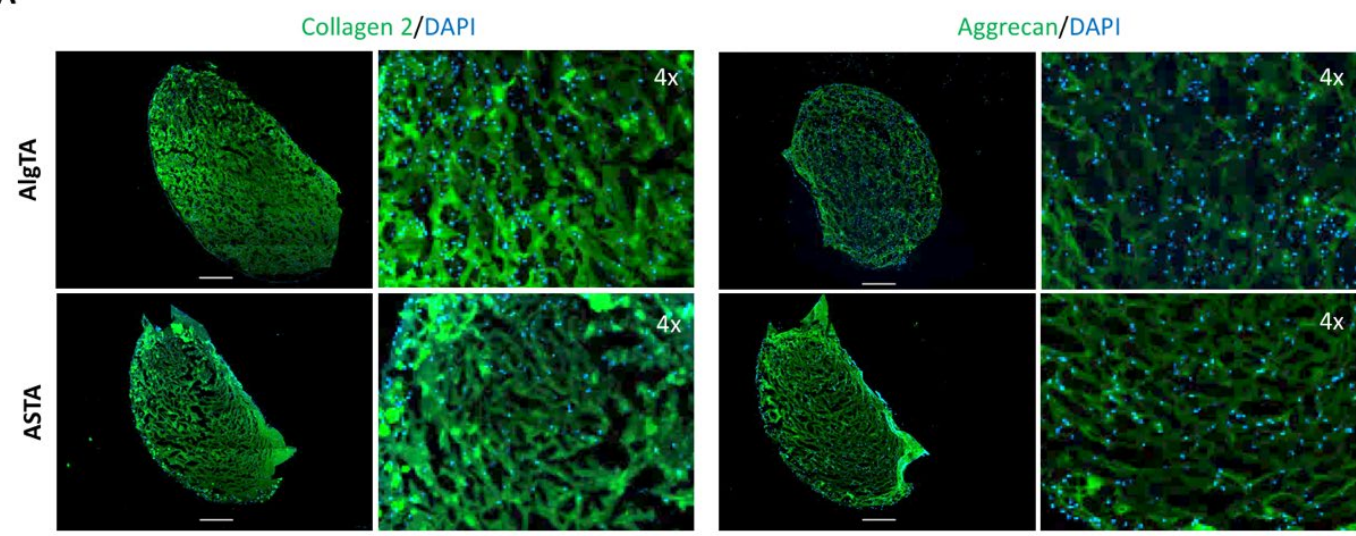

Collagen 1/DAPI

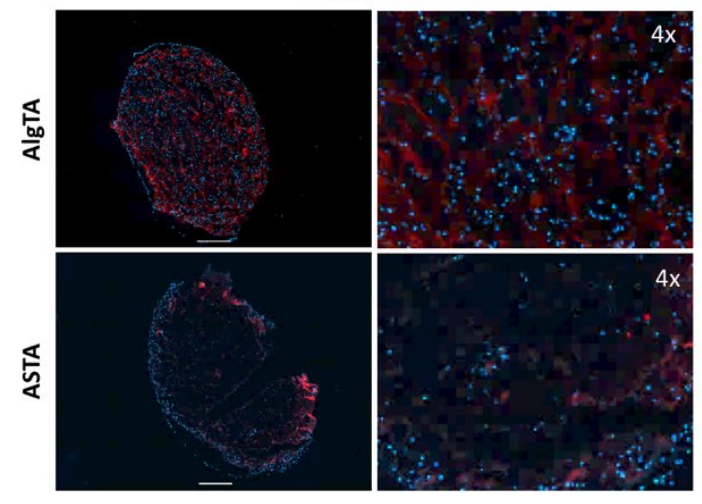

B

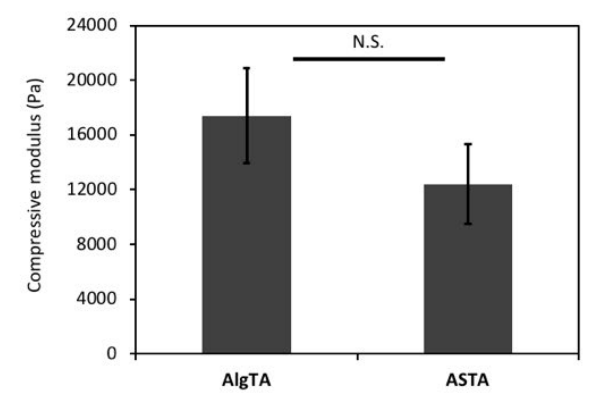

Figure 6: Cartilage matrix formation in alginate tyramine (AlgTA) and alginate sulfate tyramine (ASTA) hydrogels. (a) Immunohistochemistry images for collagen 2 (green), proteoglycan (green) and collagen 1 (red) in chondrocytes encapsulated in AlgTA and ASTA hydrogels crosslinked with tyrosinase $(1 \mathrm{kU} / \mathrm{mL})$ and cultured for 21 days in chondrogenic medium. DAPI was used as a counter-stain. Zoomed images on the right of whole gels were magnified 4 times (4x). Scale bar: $500 \mu \mathrm{m}$. (b) Compressive modulus of AlgTA and ASTA hydrogels after 21 days of culturing in chondrogenic medium expressed as mean \pm s.d. for $n=3$. N.S.: nonsignificant.

\subsection{In vivo stability of AlgTA and ASTA hydrogels and cartilage matrix formation}

AlgTA and ASTA hydrogels were implanted in subcutaneous pockets of nude mice in order to test their in vivo stability. We recently reported that ionically-crosslinked alginate sulfate hydrogels 
were not stable in vivo and dissolved soon after subcutaneous implantation (27). For more clinical relevance, we encapsulated human chondrocytes in AlgTA and ASTA hydrogels and cultured them in chondrogenic medium for 21 days. Then, the hydrogels were implanted subcutaneously into nude mice, kept for 4 weeks and removed for histological analysis and immunostainings. Both ASTA and AlgTA hydrogels were fully intact after removal from the mice. Hematoxylin \& Eosin (H\&E) staining performed on the hydrogels revealed that both hydrogels were surrounded by a thin fibrous capsule in the mice (Figure 7). Such formation of fibrous tissue has been frequently observed upon implantation of various biomaterials (44). Macroscopic signs of material toxicity in the mice such as necrosis of nearby tissue, edema or hyperemia was not observed. Furthermore, alcian blue and collagen 2 staining demonstrated deposition of cartilage-specific matrix by the human chondrocytes in the gels in vivo (Figure 7, Figure S2). Compared to in vitro control cultures in chondrogenic medium, human chondrocytes in the implanted hydrogels showed enhanced formation of collagen 2 matrix (Figure S3). Therefore, tyrosinase-mediated covalent crosslinking showed a promising method for achieving in vivo stability of alginate and alginate sulfate hydrogels while promoting cartilage matrix production in human chondrocytes. 


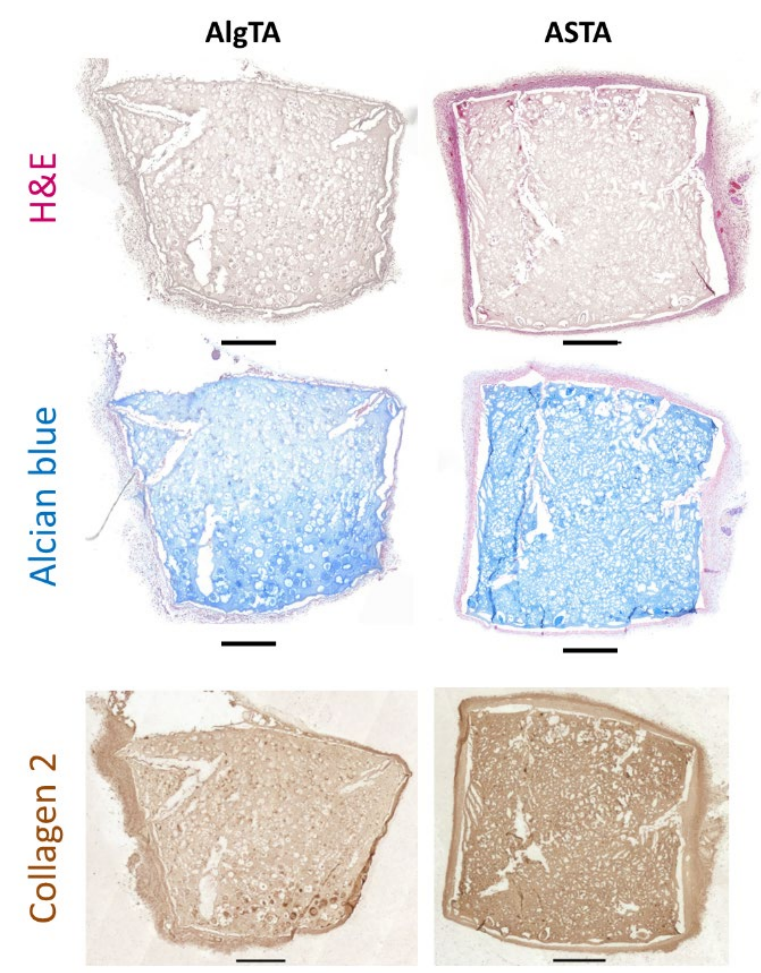

Figure 7: In vivo stability of alginate tyramine (AlgTA) and alginate sulfate tyramine (ASTA) hydrogels and cartilage matrix deposition by encapsulated human chondrocytes. Hematoxylin and eosin (H\&E), alcian blue and collagen 2 staining of AlgTA and ASTA hydrogels crosslinked with tyrosinase $(1 \mathrm{kU} / \mathrm{mL})$, cultured in vitro for 21 days followed by subcutaneous implantation in nude mice for 4 weeks. Scale bar: $500 \mu \mathrm{m}$.

\section{CONCLUSIONS}

Here, we show that double-modification of alginate with sulfate and tyramine groups enable in situ enzymatic crosslinking with tyrosinase. ASTA hydrogels provide a biomimetic and biocompatible microenvironment that support growth and re-differentiation of encapsulated bovine and human chondrocytes. Moreover, ASTA hydrogels exhibit strong adhesion to cartilage tissue through tyrosinase-mediated conjugation to tyrosine residues in the ECM collagens. Covalent crosslinking with tyrosinase also confer in vivo stability to alginate sulfate hydrogels upon subcutaneous implantation in mice and support deposition of cartilage matrix components. 
Thus, ASTA hydrogels offer an injectable, biomimetic, adhesive and stable system and demonstrate a promising potential for use in cartilage regeneration.

\section{MATERIALS AND METHODS}

Sulfation of alginates: Sulfation was performed as previously reported (28). Alginate (Pronova UP MVG, Novamatrix) (1 g) was suspended in $40 \mathrm{~mL}$ formamide (Sigma) and chlorosulfonic acid $\left(\mathrm{HClSO}_{3}\right)(99 \%$, Sigma) was added to a final concentration of $2 \% \mathrm{v} / \mathrm{v}$ in a dropwise manner on the alginate. Sulfation reaction was carried out at $60{ }^{\circ} \mathrm{C}$ with stirring for 2.5 hours. Then, cold acetone was added onto the reaction mixture and the sulfated alginate was precipitated with centrifugation at $5000 \mathrm{rpm}$ for 7 minutes. The precipitate was dissolved in deionized water and $\mathrm{pH}$ was continuously adjusted to neutral with $5 \mathrm{M} \mathrm{NaOH}$ as the sulfated alginate dissolved. The polymer was then dialyzed (12'000 Da MWCO) against $75 \mathrm{mM} \mathrm{NaCl}$ and $50 \mathrm{mM} \mathrm{NaCl}$ for 1 day each and then deionized water for 2 days followed by freeze-drying.

Characterization of sulfated alginates: Elemental analysis was performed with high-resolution inductively coupled mass spectrometry (HR-ICP-MS) on alginate dissolved in $0.1 \mathrm{M} \mathrm{HNO}_{3}$ in order to determine the sulfur content. Degree of sulfation (DS) (number of sulfate groups per alginate monomer) was estimated from the following formula assuming one sodium counterion for each negatively charged group and one water molecule per monosaccharide: Monosaccharide mass $=\mathrm{C}_{6} \mathrm{O}_{6} \mathrm{H}_{5}+(\mathrm{DS}+1) \mathrm{Na}^{+}+($DS $) \mathrm{SO}_{3}{ }^{-}+\mathrm{H}_{2} \mathrm{O}$. Size exclusion chromatography with a multiangle laser light (SEC-MALLS) detection system was used to determine the molecular weight of alginate and alginate sulfate samples. A refractive index $(\mathrm{dn} / \mathrm{dc})$ of 0.15 was used for all the polymers.

Tyramine modification of alginates: Tyramine modification was performed similarly to the previously described method for the amidation of carboxyl groups to amines via the coupling agent 4-(4,6-dimethoxy-1,3,5-triazin-2-yl)-4-methylmorpholiniumchloride (DMTMM) (29). $500 \mathrm{mg}$ 
alginate or alginate sulfate was dissolved in ultrapure water to a concentration of $1 \% \mathrm{w} / \mathrm{v}$. Tyramine hydrochloride (T2879, Sigma) and DMTMM (74104, Sigma) were added dropwise to the solution in molar equity to the carboxyl groups in alginate samples with stirring. The reaction was carried out for $72 \mathrm{~h}$ at $37^{\circ} \mathrm{C}$ and $\mathrm{pH}$ was adjusted to 6.5 at the end. The solution was dialyzed against $50 \mathrm{mM} \mathrm{NaCl}$ and then ultrapure water for 2 days each and freeze-dried. Then, a second round of tyramine modification was performed as previously reported to increase degree of modification (45). Similarly, the modified polymers were dissolved in water $(1 \% \mathrm{w} / \mathrm{v})$. Tyramine hydrochloride and DMTMM were added in molar equity to the carboxyl groups in the samples and the reaction was carried out for $24 \mathrm{~h}$ at $37{ }^{\circ} \mathrm{C}$. After the second round of modification, the samples were dialyzed again, freeze-dried and stored at $4{ }^{\circ} \mathrm{C}$ until use.

Characterization of tyramine-modified alginates: Tyramination of alginate and alginate sulfate (dissolved in deuterium oxide at a concentration of $1 \% \mathrm{w} / \mathrm{v}$ ) was characterized with ${ }^{1} \mathrm{H}$ NMR spectroscopy for the appearance of aromatic peaks at 6.85 and $7.2 \mathrm{ppm}$. Molar degree of substitution (DSmol, \%, molar ratio of conjugated tyramine residues to the total number of carboxyl groups in alginate) was assessed with UV-vis spectroscopy. A calibration curve was obtained by measuring the absorbance of solutions of tyramine hydrochloride at increasing concentrations in ultrapure water $(0.1 \mathrm{mg} / \mathrm{ml}-2 \mathrm{mg} / \mathrm{ml})$ at $275 \mathrm{~nm}$ with a plate reader (Synergy H1 Hybrid, Biotek)(30). Then, the absorbance of alginate tyramine (AlgTA) and alginate sulfate tyramine (ASTA) samples ( $1 \% \mathrm{w} / \mathrm{v}$ in ultrapure water) at $275 \mathrm{~nm}$ was measured and molar degree of substitution ( $\left.\mathrm{DS}_{\mathrm{mol}}\right)$ was calculated from the calibration curve.

Preparation of hydrogels for mechanical characterization and swelling: For hydrogel formation, polymer solutions of AlgTA and ASTA in PBS were mixed with tyrosinase (Sigma, 20kU/mL) and $25 \mu \mathrm{l}$ was pipetted into a polydimethylsiloxane (PDMS) mold with an inner diameter of $4 \mathrm{~mm}$ 
and an outer diameter of $6 \mathrm{~mm}$. A final polymer concentration of $1 \% \mathrm{w} / \mathrm{v}$ was used for AlgTA and $2 \% \mathrm{w} / \mathrm{v}$ was used for ASTA hydrogels to match the compressive moduli of the two hydrogels. The samples were incubated for 10 minutes in a humidified chamber at $37^{\circ} \mathrm{C}$ for gelation.

Compression testing of hydrogels: Compressive modulus (E) of the hydrogels was determined with a texture analyzer (Stable Micro Sytems) with a $10 \mathrm{~mm}$ measuring head and $500 \mathrm{~g}$ load cell. Compression of the samples was performed to a $10 \%$ strain at a rate of $0.01 \mathrm{~mm} / \mathrm{s}$ and the compressive modulus was calculated as the slope of the stress-strain curve in the linear viscoelastic range and expressed as mean \pm s.d. for $\mathrm{n}=3$.

Swelling of hydrogels: Hydrogels were prepared and weighed for the initial weight $\left(\mathrm{M}_{\mathrm{i}}\right)$ and incubated in PBS for 48h to reach equilibrium swelling. PBS was completely removed from the gels and the gels were weighed again after swelling $\left(\mathrm{M}_{\mathrm{S}}\right)$. Then the gels were freeze-dried and their dry weights $\left(\mathrm{M}_{\mathrm{D}}\right)$ were obtained. Swelling ratio was expressed as percent change in dry mass $(\%)$ $\left(\mathrm{M}_{\mathrm{S}}-\mathrm{M}_{\mathrm{D}} / \mathrm{M}_{\mathrm{D}}\right)$ and as mean \pm s.d. for $\mathrm{n}=5$.

Push-out test: Bovine articular cartilage pieces with 1-2 mm thickness were removed with a scalpel. Cartilage rings with an outer diameter of $8 \mathrm{~mm}$ and an inner diameter of $4 \mathrm{~mm}$ were punched out from the cartilage and washed in PBS. Then, the inner parts of the rings were digested with $1 \mathrm{U} / \mathrm{mL}$ chondroitinase $\mathrm{ABC}$ (Sigma) for 15 minutes at $37^{\circ} \mathrm{C}$ and washed several times with PBS. Enzymatically crosslinked hydrogels were directly casted in the cartilage rings by mixing the ASTA and AlgTA polymer solutions and tyrosinase (final concentration of $1 \mathrm{kU} / \mathrm{ml}$ ) and incubated for 30 minutes. For calcium-crosslinked hydrogels, polymer solutions were pipetted in the cartilage ring, which was covered with $100 \mathrm{mM} \mathrm{CaCl}_{2}$ solution and allowed to gel for 30 minutes. Push-out tests were performed using a $3 \mathrm{~mm}$ cylindrical plate to push the sample at a 
speed of $0.5 \mathrm{~mm} / \mathrm{s}$. Bond strength was expressed as the maximum force measured divided by the integration area in the inner part of the gel and as mean \pm s.d. for $n=3$.

Bovine chondrocyte isolation: Articular cartilage was removed from the condyles of cow (1-2 years old) knees with a sterile blade, minced into small pieces and washed with DMEM (Glutamax, high glucose, $4.5 \mathrm{~g} / \mathrm{L}$ glucose) (Life Technologies) supplemented with 1\% penicillin-streptomycin $(\mathrm{P} / \mathrm{S})($ Gibco). Cartilage pieces were digested with $0.1 \%$ collagenase (Sigma) in DMEM supplemented with $5 \%$ fetal bovine serum (FBS) (Life Technologies) for $5 \mathrm{~h}$ at $37{ }^{\circ} \mathrm{C}$ with gentle agitation. Digested cartilage was sieved with a $100 \mu \mathrm{m}$ and then a $40 \mu \mathrm{m}$ cell strainer and centrifuged at $500 \mathrm{~g}$ for $10 \mathrm{~min}$. The chondrocyte pellet was then washed twice with growth medium (DMEM containing 10\% FBS and $50 \mu \mathrm{g} / \mathrm{mL}$ L-Ascorbic acid -2-phosphate (Sigma)). Isolated chondrocytes were seeded at a density of $3000 \mathrm{cells} / \mathrm{cm}^{2}$ and passaged at $80-90 \%$ confluency until passage 1 before encapsulation in hydrogels.

Human chondrocyte isolation: Non-arthritic articular cartilage samples were obtained from surgical knee operations (ethics approval number KEK-ZH 2013-0097). The cartilage samples were minced with a sterile blade into $1-3 \mathrm{~mm}^{3}$ pieces and washed with DMEM (high glucose) supplemented with $10 \mu \mathrm{g} / \mathrm{mL}$ gentamycin. The cartilage pieces were digested with $0.1 \%$ collagenase (Sigma) in DMEM supplemented with 10\% fetal bovine serum (FBS) (Invitrogen) overnight at $30{ }^{\circ} \mathrm{C}$ with gentle shaking and sieved through a $100 \mu \mathrm{m}$ and then a $40 \mu \mathrm{m}$ cell strainer. The chondrocytes were centrifuged at $500 \mathrm{~g}$ for $10 \mathrm{~min}$ and washed twice with growth medium. The chondrocytes were seeded at a density of $3000 \mathrm{cells} / \mathrm{cm}^{2}$ and expanded to passage 2 before encapsulation into hydrogels. 
Encapsulation of chondrocytes in hydrogels and culturing: AlgTA and ASTA were dissolved in phenol-free DMEM (high glucose) and sterile filtered ( $0.2 \mu \mathrm{m}$ pore size). The polymer solutions (final concentration of $2 \% \mathrm{w} / \mathrm{v}$ for ASTA and $1 \% \mathrm{w} / \mathrm{v}$ for AlgTA) then were mixed with the chondrocyte suspension (final cell density of $10^{7}$ cells $/ \mathrm{mL}$ ) and tyrosinase (final concentration of $1 \mathrm{kU} / \mathrm{mL}$ ). $25 \mu 1$ were pipetted on PDMS molds and incubated for 30 minutes in a humidified chamber at $37{ }^{\circ} \mathrm{C}$ for gelation. Chondrocytes encapsulated in hydrogels were cultured in chondrogenic medium containing phenol-free DMEM (high glucose) supplemented with 10 $\mathrm{ng} / \mathrm{mL}$ transforming growth factor $\beta 3$ (TGF- $\beta 3$, Peprotech), $100 \mathrm{nM}$ dexamethasone, $50 \mu \mathrm{g} / \mathrm{mL} \mathrm{L}$ ascorbate-2-phosphate, $40 \mu \mathrm{g} / \mathrm{mL}$ L-proline, 1\% P/S, and 1\% ITS + Premix (Corning). The hydrogels were cultured at $5 \% \mathrm{CO}_{2}$ and $37{ }^{\circ} \mathrm{C}$ for 3 weeks and medium was changed 3 times a week.

Assessment of chondrocyte viability: Cell viability was assessed with calcein AM and propidium iodide (PI) staining. The hydrogels were incubated in culture medium with $2 \mu \mathrm{M}$ calcein AM 30 $\mu \mathrm{g} / \mathrm{mL}$ PI for 1 hour at $37^{\circ} \mathrm{C}$ followed by washing with growth medium twice for 15 minutes and imaging with fluorescence microscopy (Zeiss Axio Observer). For the quantification of viability, $200 \mu \mathrm{m}$ z-stacks from the gels were projected with maximum intensity followed by determination of the number of live and dead cells with ImageJ.

Real-time PCR: Hydrogels were collected after 3 weeks of culturing and frozen in liquid nitrogen. The hydrogels were then homogenized with an electrical tissue pestle in Trizol ${ }^{\circledR}$ (Life Technologies) and centrifuged at $12^{\prime} 000 \mathrm{~g}$ for $10 \mathrm{~min}$ at $4^{\circ} \mathrm{C}$. The supernatant was removed and phase-separated with chloroform. The aqueous phase was taken carefully after centrifugation at $12^{\prime} 000 \mathrm{~g}$ for $15 \mathrm{~min}$ at $4^{\circ} \mathrm{C}$. RNA isolation was then performed using the NucleoSpin miRNA kit (Macherey-Nagel AG) according to the manufacturer's instructions. For the 2D samples, cell lysis 
buffer (Macherey-Nagel AG) was directly pipetted on the cells and the lysate was removed with a scraper. RNA isolation was similarly performed with the NucleoSpin miRNA kit. RNA concentration of the samples was quantified with a plate reader (Tek3 plate, Synergy, BioTek, Inc.). RNA was reverse transcribed using SuperScript III reverse transcriptase (Life Technologies) and cDNA was amplified by quantitative real-time PCR (StepOnePlus, Applied Biosystems). Ribosomal protein L13 (RPL13a) was used as an internal reference gene and fold change was quantified with the $\Delta \Delta \mathrm{Ct}$ method. The following primers for bovine (Microsynth $\mathrm{AG}$ ) were used in this study: RPL13a (forward (F) 5'- GCCAAGATCCACTATCGGAAA-3'; reverse (R) 5'AGGACCTCTGTGAATTTGCC-3'), COL2A1 (Col2) (F, 5'-GGCCAGCGTCCCCAAGAA-3'; R, 5'-AGCAGGCGCAGGAAGGTCAT-3'), ACAN (aggrecan) (F, 5'GGGAGGAGACGACTGCAATC-3'; R， 5'-CCCATTCCGTCTTGTTTTCTG-3')， COL1A2 (Col1) (F, 5'-CGAGGGCAACAGCAGATTCACTTA-3'; $\quad$ R, $\quad$ 5'GCAGGCGAGATGGCTTGTTTG-3'), SOX9 (F, 5'-ACGCGGCCC CAGGAGAAC-3'; R, 5'CGGATGCACACGGGGAACTT-3'), MMP13 (F, 5'-AAACATCCCAAAACGCCAGACAA3'; R, 5'-AGGATGCAGCCG CCAGAAGA-3'), ADAMTS5 (F, 5'GATGGTCACGGTAACTGTTTGCT-3'; R, 5'-GCCGGGACACACCGAGTAC-3').

Immunohistochemisty: Hydrogels were fixed with $4 \%$ formaldehyde (Sigma) with $0.1 \%$ Triton-X (Sigma) in PBS for $1 \mathrm{~h}$ at $4{ }^{\circ} \mathrm{C}$ and washed again with PBS. Hydrogels were embedded in optimum cutting temperature compound (OCT, VWR) overnight, snap-frozen and cut into $5 \mu \mathrm{m}$ sections with a cryotome (CryoStar NX70, ThermoScientific). The sections were washed with PBS to remove the OCT. For collagen 2 staining, epitope retrieval was performed by incubating the sections in $2 \mathrm{mg} / \mathrm{mL}$ hyaluronidase (Sigma) in PBS for $30 \mathrm{~min}$ at $37^{\circ} \mathrm{C}$. For the proteoglycan staining, the sections were incubated with $10 \mathrm{mM}$ dithiothreitol (DTT) (Sigma) in $50 \mathrm{mM}$ Tris- 
$\mathrm{HCl}$ and $200 \mathrm{mM} \mathrm{NaCl}(\mathrm{pH} 7.4)$ for $2 \mathrm{~h}$ at $37^{\circ} \mathrm{C}$ and then alkylated with $40 \mathrm{mM}$ iodoacetamide in PBS for $1 \mathrm{~h}$ at $37^{\circ} \mathrm{C}$. Then, the sections were digested with chondroitinase $\mathrm{ABC}(0.2 \mathrm{U} / \mathrm{mL})$ (Sigma) for $20 \mathrm{~min}$ at $37^{\circ} \mathrm{C}$. The sections were then blocked with 5\% BSA for $1 \mathrm{~h}$ at RT followed by incubation with primary antibody in $1 \%$ BSA overnight at $4{ }^{\circ} \mathrm{C}$. After primary antibody incubation, the sections were washed three times in PBS, incubated with the secondary antibody in 1\% BSA (IgG goat antimouse AlexaFluor 594, Invitrogen) for $1 \mathrm{~h}$ at RT, washed again and incubated with phalloidin-rhodamine and DAPI in PBS for $15 \mathrm{~min}$ at RT. Then, the sections were covered with a coverslip with aqueous mounting media (Vector Laboratories) and imaged with fluorescence microscopy. The primary antibodies used were anti-collagen 2 (Rockland, 600-401104-0.5), anti-proteoglycan hyaluronic acid-binding region (12/21/1-C-6, DSHB), anti-collagen 1 (Abcam, ab6308).

Subcutaneous implantation of hydrogels: AlgTA $(1 \% \mathrm{w} / \mathrm{v})$ and ASTA $(2 \% \mathrm{w} / \mathrm{v})$ hydrogels were prepared with $1 \mathrm{kU} / \mathrm{mL}$ tyrosinase with human chondrocytes at a density of $10^{7}$ cells $/ \mathrm{mL}$. The hydrogels were pre-cultured in chondrogenic medium for 3 weeks and implanted in the subcutaneous pocket of NU/NU nude mice (2-3 month-old female mice, Charles River). Animal experimentation was performed in accordance with the ethical guidelines (Licence number: ZH189/2014). The mice were initially anesthetized with $4.5 \%$ isofluorane and during the surgery $2 \%$ isoflurane was applied via a nose mask. Two incisions were made in each mouse, the hydrogels were placed subcutaneously and closed with surgical staples. After 4 weeks, the animals were euthanized via $\mathrm{CO}_{2}$ asphyxiation and the hydrogels were removed.

Histological stainings: Hydrogels were fixed with $4 \%$ formaldehyde (Sigma) with $0.1 \%$ Triton-X (Sigma) in PBS for $1 \mathrm{~h}$ at $4{ }^{\circ} \mathrm{C}$ and washed again with PBS. Hydrogels were embedded in OCT overnight, snap-frozen and cut into $5 \mu \mathrm{m}$ sections with a cryotome (CryoStar NX70, 
ThermoScientific). The sections were washed with PBS to remove the OCT. For the Hematoxylin \& eosin $(\mathrm{H} \& \mathrm{E})$ staining, the sections were incubated with Harris Hematoxylin solution (Thermo Scientific) for $2 \mathrm{~min}$, washed with $\mathrm{H}_{2} \mathrm{O}$ for 5 min, incubated in clarifier reagent (Thermo Scientific) for $2 \mathrm{~min}$ and washed with $\mathrm{H}_{2} \mathrm{O}$ for $1 \mathrm{~min}$. The sections were then exposed to bluing agent (Thermo scientific), washed with $\mathrm{H}_{2} \mathrm{O}$ for $5 \mathrm{~min}$, rinsed in $95 \%$ alcohol and counterstained briefly in $0.25 \%$ (v/v) eosin $\mathrm{Y}$ (Sigma) solution in $70 \%$ ethanol. After washing extensively with $\mathrm{H}_{2} \mathrm{O}$, the sections were dehydrated with $95 \%$ and $100 \%$ ethanol respectively and were mounted with hardening medium. For the alcian blue staining, the sections were washed with double-distilled $\mathrm{H}_{2} \mathrm{O}\left(\mathrm{ddH}_{2} \mathrm{O}\right)$ and incubated with 3\% acetic acid (Sigma) for $10 \mathrm{~min}$. Then, the samples were incubated with alcian blue solution (Sigma, $1 \%$ in 3\% acetic acid) for 20 min, washed with $\mathrm{ddH}_{2} \mathrm{O}$ and mounted with hardening medium. Mounted samples were imaged with an automated slide scanner (Panoramic 250 Flash II, 3Dhistech).

Colorimetric collagen 2 immunostaining: Epitope retrieval was performed by incubating the sections in $2 \mathrm{mg} / \mathrm{mL}$ hyaluronidase (Sigma) in TBS with $1 \% \mathrm{BSA}$ for $30 \mathrm{~min}$ at $37^{\circ} \mathrm{C}$. The sections were washed twice in TBS with $0.025 \%$ Triton X-100 for $5 \mathrm{~min}$. The sections were then blocked with $5 \%$ BSA for $1 \mathrm{~h}$ at RT followed by incubation with primary antibody (anti collagen 2, Rockland, 600-401-104-0.5) in TBS with $1 \%$ BSA overnight at $4{ }^{\circ} \mathrm{C}$. Next, the sections were rinced twice in TBS with $0.025 \%$ Triton- $X$ for 5 min and the endogenous peroxidase activity was blocked by treating the samples with $0.3 \% \mathrm{H}_{2} \mathrm{O}_{2}$ for 30 min at RT. After washing three times in TBS for $5 \mathrm{~min}$, the sections were incubated with the secondary antibody (goat anti-rabbit IgGHRP, Abcam ab6721) in TBS with 1\% BSA for 1 hour at RT. The samples were rinsed with TBS three times for $5 \mathrm{~min}$. Then, the colorimetric reaction was performed using the DAB Substrate kit (ab64238) and the DAB enhancer (Abcam, ab64238) according to the manufacturer's instructions. 
The sections were rinsed with $\mathrm{H}_{2} \mathrm{O}$ for 5 minutes, dehydrated in sequential ethanol bathes $(50 \%$, $70 \%, 95 \%$ and $100 \%$ ) for $5 \mathrm{~min}$ and incubated with xylene twice for $3 \mathrm{~min}$. The slides were then mounted with hardening medium and imaged with an automated slide scanner (Panoramic 250 Flash II, 3Dhistech).

Statistical analysis: Quantitative data was expressed as mean \pm standard deviation (s.d.). Statistical analyses were carried out with OriginPro 9.1 by analysis of variance (ANOVA) with Tukey's and Bonferonni's post-hoc tests for multiple comparisons and $\mathrm{p}$ values less than 0.05 were considered as statistically significant.

\section{AUTHOR INFORMATION}

\section{Corresponding Author}

Marcy Zenobi-Wong, PhD

E-mail: zmarcy@ethz.ch

biofabrication.ethz.ch

ETH Zurich, HPL J22, Otto-Stern-Weg 7

CH-8093 Zurich, Switzerland

\section{Author Contributions}

E.O. conceptualized the project. E.O. and M.Z.W. designed experiments. E.O., C.L., T.S., E.C., Ø.A. conducted experiments and analyzed data. E.O. wrote the manuscript. All authors have given approval to the final version of the manuscript.

\section{Funding Sources}

This work was funded by Swiss National Science Foundation (315230_159783 and 315230_143667) and Center for Applied Biotechnology and Molecular Medicine (CABMM).

\section{Notes}

The authors declare no competing interests. 


\section{REFERENCES}

1. Makris EA, Gomoll AH, Malizos KN, Hu JC, Athanasiou KA. Repair and tissue engineering techniques for articular cartilage. Nature reviews Rheumatology. 2015;11(1):21-34.

2. Darling EM, Athanasiou KA. Rapid phenotypic changes in passaged articular chondrocyte subpopulations. Journal of orthopaedic research : official publication of the Orthopaedic Research Society. 2005;23(2):425-32.

3. Knudson CB, Knudson W. Cartilage proteoglycans. Seminars in cell \& developmental biology. 2001;12(2):69-78.

4. Chen G, Sato T, Ushida T, Hirochika R, Tateishi T. Redifferentiation of dedifferentiated bovine chondrocytes when cultured in vitro in a PLGA-collagen hybrid mesh. FEBS letters. 2003;542(1-3):95-9.

5. Benya PD, Shaffer JD. Dedifferentiated chondrocytes reexpress the differentiated collagen phenotype when cultured in agarose gels. Cell. 1982;30(1):215-24.

6. Hauselmann HJ, Fernandes RJ, Mok SS, Schmid TM, Block JA, Aydelotte MB, et al. Phenotypic stability of bovine articular chondrocytes after long-term culture in alginate beads. Journal of cell science. 1994;107 ( Pt 1):17-27.

7. Li Y, Rodrigues J, Tomas H. Injectable and biodegradable hydrogels: gelation, biodegradation and biomedical applications. Chemical Society reviews. 2012;41(6):2193-221.

8. Kurisawa M, Chung JE, Yang YY, Gao SJ, Uyama H. Injectable biodegradable hydrogels composed of hyaluronic acid-tyramine conjugates for drug delivery and tissue engineering. Chem Commun (Camb). 2005(34):4312-4.

9. Yanga J, Yeom J, Hwang BW, Hoffman AS, Hahn SK. In situ-forming injectable hydrogels for regenerative medicine. Progress in polymer science. 2014;39(12):1973-86. 10. Moreira Teixeira LS, Bijl S, Pully VV, Otto C, Jin R, Feijen J, et al. Self-attaching and cell-attracting in-situ forming dextran-tyramine conjugates hydrogels for arthroscopic cartilage repair. Biomaterials. 2012;33(11):3164-74.

11. Jin R, Moreira Teixeira LS, Dijkstra PJ, van Blitterswijk CA, Karperien M, Feijen J. Chondrogenesis in injectable enzymatically crosslinked heparin/dextran hydrogels. Journal of controlled release : official journal of the Controlled Release Society. 2011;152(1):186-95.

12. Jin R, Teixeira LS, Dijkstra PJ, van Blitterswijk CA, Karperien M, Feijen J.

Enzymatically-crosslinked injectable hydrogels based on biomimetic dextran-hyaluronic acid conjugates for cartilage tissue engineering. Biomaterials. 2010;31(11):3103-13.

13. Broguiere N, Cavalli E, Salzmann GM, Applegate LA, Zenobi-Wong M. Factor XIII Cross-Linked Hyaluronan Hydrogels for Cartilage Tissue Engineering. ACS Biomaterials Science \& Engineering. 2016;2(12):2176-84.

14. Wang R, Leber N, Buhl C, Verdonschot N, Dijkstra PJ, Karperien M. Cartilage adhesive and mechanical properties of enzymatically crosslinked polysaccharide tyramine conjugate hydrogels. Polymers for Advanced Technologies. 2014;25(5):568-74.

15. Chen T, Embree HD, Wu LQ, Payne GF. In vitro protein-polysaccharide conjugation: tyrosinase-catalyzed conjugation of gelatin and chitosan. Biopolymers. 2002;64(6):292-302.

16. Chen T, Embree HD, Brown EM, Taylor MM, Payne GF. Enzyme-catalyzed gel formation of gelatin and chitosan: potential for in situ applications. Biomaterials.

2003;24(17):2831-41. 
17. Anghileri A, Lantto R, Kruus K, Arosio C, Freddi G. Tyrosinase-catalyzed grafting of sericin peptides onto chitosan and production of protein-polysaccharide bioconjugates. Journal of biotechnology. 2007;127(3):508-19.

18. Demolliens A, Boucher C, Durocher Y, Jolicoeur M, Buschmann MD, De Crescenzo G. Tyrosinase-catalyzed synthesis of a universal coil-chitosan bioconjugate for protein immobilization. Bioconjugate chemistry. 2008;19(9):1849-54.

19. Kang GD, Lee KH, Ki CS, Nahm JH, Park YH. Silk fibroin/chitosan conjugate crosslinked by tyrosinase. Macromolecular Research. 2004;12(5):534-9.

20. Lee BP, Dalsin JL, Messersmith PB. Synthesis and gelation of DOPA-modified poly(ethylene glycol) hydrogels. Biomacromolecules. 2002;3(5):1038-47.

21. Yamada K, Chen T, Kumar G, Vesnovsky O, Topoleski LD, Payne GF. Chitosan based water-resistant adhesive. Analogy to mussel glue. Biomacromolecules. 2000;1(2):252-8.

22. Kim SH, An YH, Kim HD, Kim K, Lee SH, Yim HG, et al. Enzyme-mediated tissue adhesive hydrogels for meniscus repair. Int J Biol Macromol. 2018;110:479-87.

23. Kim SH, Lee SH, Lee JE, Park SJ, Kim K, Kim IS, et al. Tissue adhesive, rapid forming, and sprayable ECM hydrogel via recombinant tyrosinase crosslinking. Biomaterials. 2018;178:401-12.

24. Liu HY, Greene T, Lin TY, Dawes CS, Korc M, Lin CC. Enzyme-mediated stiffening hydrogels for probing activation of pancreatic stellate cells. Acta biomaterialia. 2017;48:258-69. 25. Liu HY, Korc M, Lin CC. Biomimetic and enzyme-responsive dynamic hydrogels for studying cell-matrix interactions in pancreatic ductal adenocarcinoma. Biomaterials. 2018;160:24-36.

26. Öztürk E, Arlov Ø, Aksel S, Li L, Ornitz DM, Skjåk-Bræk G, et al. Sulfated Hydrogel Matrices Direct Mitogenicity and Maintenance of Chondrocyte Phenotype through Activation of FGF Signaling. Advanced Functional Materials. 2016;26(21):3649-62.

27. Formica FA, Ozturk E, Hess SC, Stark WJ, Maniura-Weber K, Rottmar M, et al. A Bioinspired Ultraporous Nanofiber-Hydrogel Mimic of the Cartilage Extracellular Matrix. Advanced healthcare materials. 2016;5(24):3129-38.

28. Arlov O, Aachmann FL, Sundan A, Espevik T, Skjak-Braek G. Heparin-like properties of sulfated alginates with defined sequences and sulfation degrees. Biomacromolecules. 2014;15(7):2744-50.

29. Loebel C, D'Este M, Alini M, Zenobi-Wong M, Eglin D. Precise tailoring of tyraminebased hyaluronan hydrogel properties using DMTMM conjugation. Carbohydrate polymers. 2015;115:325-33.

30. Darr A, Calabro A. Synthesis and characterization of tyramine-based hyaluronan hydrogels. Journal of materials science Materials in medicine. 2009;20(1):33-44.

31. Wu LQ, Bentley WE, Payne GF. Biofabrication with biopolymers and enzymes: potential for constructing scaffolds from soft matter. The International journal of artificial organs. 2011;34(2):215-24.

32. Jin R, Lou B, Lin C. Tyrosinase-mediated in situ forming hydrogels from biodegradable chondroitin sulfate-tyramine conjugates. Polymer International. 2013;62(3):353-61.

33. Das S, Pati F, Choi YJ, Rijal G, Shim JH, Kim SW, et al. Bioprintable, cell-laden silk fibroin-gelatin hydrogel supporting multilineage differentiation of stem cells for fabrication of three-dimensional tissue constructs. Acta biomaterialia. 2015;11:233-46. 
34. Kim MK, Choi SW, Kim SR, Oh IS, Won MH. Autologous chondrocyte implantation in the knee using fibrin. Knee surgery, sports traumatology, arthroscopy : official journal of the ESSKA. 2010;18(4):528-34.

35. Patel S, Rodriguez-Merchan EC, Haddad FS. The use of fibrin glue in surgery of the knee. The Journal of bone and joint surgery British volume. 2010;92(10):1325-31.

36. Wang DA, Varghese S, Sharma B, Strehin I, Fermanian S, Gorham J, et al. Multifunctional chondroitin sulphate for cartilage tissue-biomaterial integration. Nature materials. 2007;6(5):385-92.

37. Sharma B, Fermanian S, Gibson M, Unterman S, Herzka DA, Cascio B, et al. Human cartilage repair with a photoreactive adhesive-hydrogel composite. Science translational medicine. 2013;5(167):167ra6.

38. Rowley JA, Madlambayan G, Mooney DJ. Alginate hydrogels as synthetic extracellular matrix materials. Biomaterials. 1999;20(1):45-53.

39. Balakrishnan B, Joshi N, Jayakrishnan A, Banerjee R. Self-crosslinked oxidized alginate/gelatin hydrogel as injectable, adhesive biomimetic scaffolds for cartilage regeneration. Acta biomaterialia. 2014;10(8):3650-63 .

40. Jenkins CL, Meredith HJ, Wilker JJ. Molecular weight effects upon the adhesive bonding of a mussel mimetic polymer. ACS applied materials \& interfaces. 2013;5(11):5091-6.

41. Leivonen SK, Chantry A, Hakkinen L, Han J, Kahari VM. Smad3 mediates transforming growth factor-beta-induced collagenase-3 (matrix metalloproteinase-13) expression in human gingival fibroblasts. Evidence for cross-talk between Smad3 and p38 signaling pathways. The Journal of biological chemistry. 2002;277(48):46338-46.

42. Ravanti L, Hakkinen L, Larjava H, Saarialho-Kere U, Foschi M, Han J, et al.

Transforming growth factor-beta induces collagenase-3 expression by human gingival fibroblasts via p38 mitogen-activated protein kinase. The Journal of biological chemistry. 1999;274(52):37292-300.

43. Freeman I, Kedem A, Cohen S. The effect of sulfation of alginate hydrogels on the specific binding and controlled release of heparin-binding proteins. Biomaterials. 2008;29(22):3260-8.

44. Park KM, Lee Y, Son JY, Oh DH, Lee JS, Park KD. Synthesis and characterizations of in situ cross-linkable gelatin and 4-arm-PPO-PEO hybrid hydrogels via enzymatic reaction for tissue regenerative medicine. Biomacromolecules. 2012;13(3):604-11.

45. Loebel C, Stauber T, D'Este M, Alini M, Zenobi-Wong M, Eglin D. Fabrication of cellcompatible hyaluronan hydrogels with a wide range of biophysical properties through high tyramine functionalization. Journal of Materials Chemistry B. 2017;5(12):2355-63. 